\title{
'Can you hear me?': communication, relationship and ethics in video-based telepsychiatric consultations
}

\author{
Eva-Maria Frittgen, ${ }^{1}$ Joschka Haltaufderheide ${ }^{2}$
}

${ }^{1}$ Center for Psychiatry Reichenau, Reichenau, BadenWürttemberg, Germany ${ }^{2}$ Institute for Medical Ethics and History of Medicine, Ruhr University Bochum, Bochum, Germany

\section{Correspondence to}

Dr. Eva-Maria Frittgen, Center for Psychiatry Reichenau, 78479 Reichenau, Baden-Württemberg, Germany;

e.frittgen@zfp-reichenau.de

E-MF and JH contributed equally.

Received 24 March 2021 Accepted 4 September 2021

\section{Check for updates}

(c) Author(s) (or their employer(s)) 2021. No commercial re-use. See rights and permissions. Published by BMJ.

To cite: Frittgen E-M, Haltaufderheide J.

$J$ Med Ethics Epub ahead of print: [please include Day

Month Year]. doi:10.1136/

medethics-2021-107434

\section{ABSTRACT}

Telepsychiatry has long been discussed as a supplement to or substitute for face-to-face therapeutic consultations. The current pandemic crisis has fueled the development in an unprecedented way. More and more psychiatric consultations are now carried out online as video-based consultations. Treatment results appear to be comparable with those of face-to-face care in terms of clinical outcome, acceptance, adherence and patient satisfaction. However, evidence on videoconferencing in a variety of different fields indicates that there are extensive changes in the communication behaviour in online conversations. We hypothesise that this might impact ethically relevant aspects of the therapeutic relationship, which plays a prominent role in psychiatry. In this paper, we review effects of video-based consultations on communication between therapists and patients in psychiatry. Based on a common understanding of video-based consultations as changing the lived experience of communication, we categorise these effects according to sensory, spatial and technical aspects. Departing from a power-based model of therapeutic relationships, we then discuss the ethical significance of this changed communication situation, based on dimensions of respect for autonomy, lucidity, fidelity, justice and humanity. We conclude that there is evidence for ethically relevant changes of the therapeutic relationship in video-based telepsychiatric consultations. These changes need to be more carefully considered in psychiatric practice and future studies.

\section{BACKGROUND}

Digitised healthcare has received an enormous impulse due to the impact of the COVID-19 pandemic. Among telepsychiatry, videoconferencing is one of the most common forms of communication. ${ }^{1}$ It has been used in practice and is discussed in the scientific community for more than 60 years. ${ }^{1}$ It is expected to play an important role in the future of psychiatry $^{2}$ as the physical examination of patients takes up less space than in other disciplines and communication with patients is given more weight. Given the current situation, video-based consultation seems to be a way to ensure patient care while avoiding the risk of infection. ${ }^{3}$

Empirical studies suggest that video-based consultation in psychiatry can be used in evaluation, diagnosis, treatment and education ${ }^{4}$ and without restriction for all psychiatric disorders. ${ }^{5}$ Treatment results seem to be comparable to face-to-face care, indicating positive effects, especially regarding the quality and continuity of care and coverage of rural areas with mental health services. ${ }^{467}$

Often noted, but rarely the subject of investigation, are, however, factors that elude quantifiability and measurability, such as the ethical quality of treatment or individual characteristics of the therapeutic alliance. ${ }^{8-10}$ These include impact on the roles of patients and health professionals or the significance of changes in communication patterns between both parties and the significance of in-person contact. ${ }^{8}$

All of the factors above are important elements in the therapeutic relationship. The influence of video-based consultation on patients and therapists' perception and satisfaction with the therapeutic alliance has been investigated in a number of studies,${ }^{6811}$ mainly from the perspective of clinical outcomes. Surveys and standardised measures indicate hardly any difference from face-to-face care, especially from the patients' point of view. ${ }^{811}$ Communicational drawbacks, such as the lack of physical proximity and nonverbal cues in videoconferencing, are frequently considered as possible hurdles. ${ }^{12}$ However, studies relating to the influence on the therapeutic relationship and analysing the ethical significance of changes are rare. ${ }^{613-15}$

The therapeutic relationship is of special significance from an ethical point of view. It is considered to be worthy of protection and, in addition, has shown to be a decisive factor for treatment outcome. ${ }^{16}$ The impact of video-based consultation needs to be carefully analysed. ${ }^{17}$ In this paper, we seek to answer the question whether video-based consultation can affect ethically relevant dimensions of the therapeutic relationship and, if so, how this should be addressed. Therefore, we investigate the effects of video-based consultation on the therapeutic relationship in psychiatry from an empirical and ethical perspective. First, we discuss the normative foundations of a meaningful therapeutic relationship. In particular, we elaborate on the ethically required distribution of rights and duties between professionals and patients and certain implications for behaviour in communication situations that arise directly from these demands. Second, we review and categorise the empirical effects of video-based consultations and the changes in communication between professionals and patients in psychiatry. Finally, we discuss the ethical significance of these changes by examining some exemplary points which specially consider the therapeutic relationship.

\section{ETHICAL DIMENSIONS OF THE THERAPEUTIC RELATIONSHIP IN PSYCHIATRY}

Traditional approaches to the therapeutic relationship in medical ethics usually define a list of principles or duties to fulfil. However, modern approaches have sought to develop more fine-grained accounts which are able to theoretically ground and conceptualise rights and obligations. ${ }^{18} 19$ A common way 
to ethically outline therapeutic relationships in this regard is by referring to its communicative and social underpinnings. In this view, a help-seeking person and their caregiving professional enter into a relationship which can be characterised by its imbalance regarding different dimensions of power. ${ }^{19}{ }^{20}$ Power, as the ability to decide and act, emerges from the unequal distribution of force, material resources and knowledge as determined by the social, cultural and institutional context of a situation..$^{22}$ Shaping this relationship according to a moral standard, hence, requires the development of a set of different rights and obligations for both parties based on an understanding of what values ought to be protected.

While earlier models understood the imbalance between patients and professionals to be justified by the professionals' duty to beneficence and accepted diminished patient autonomy, the last decades have brought a decisive change in social determinants (eg, increased access to information, changed exclusive status of professionals) and value considerations. Nowadays, contemporary models strongly emphasise the respect for autonomy and seek to establish a balance between professionals' duties to beneficence and avoidance of harm, on the one hand, and patients' self-determination, on the other. The requirement of this balance obliges patients and professionals to engage in a relationship that is grounded in respect for autonomy, lucidity, fidelity, justice and humanity towards each other. ${ }^{23} 24$

These terms loosely refer to Brody ${ }^{18}$ and Fried ${ }^{25}$ and do not depict fully formulated rights in themselves but rather present categories of meaningful relationships from an ethical perspective. Respect for autonomy, as a primary cornerstone, includes understanding individuals as being capable of self-legislation, that is, being able to decide voluntarily to act on principles that meet or flout general principles of thought and have a reason to act according to the former. ${ }^{26}$ This includes positive rights to the assistance in or fostering of autonomous decision-making and negative rights, for example, to refuse further treatment. ${ }^{27}$ Rights regarding lucidity, fidelity, justice and humanity and their respective duties are of instrumental value in creating and maintaining conditions that allow for autonomy. ${ }^{20}$ The dimension of lucidity entails rights and duties to clarity and transparency that account for a truthful and open communication. Regarding clarity, this includes refraining from knowingly communicating false information and deception. ${ }^{23}$ Second, it requires the clear, comprehensive and accurate transmission of information and communication. ${ }^{28}$ Transparency, on the other hand, obliges both parties to disclosure of all the information necessary to allow for autonomous decision making. On the side of therapists, a full disclosure of pertinent information, for example, on treatment, prognosis and alternatives, enables patients to make informed choices of their own accord which are covered by a person's right to autonomy. On the side of the patients, honest and truthful disclosure of experiences of illness is essential for diagnosis and treatment. ${ }^{24}$

Fidelity, as the second dimension, entails rights and duties regarding stability, durability, accountability and confidentiality. Issues of fidelity are known to arise whenever individuals voluntarily enter into a relationship. By consenting to such a relationship, an ethical commitment to its boundaries is implied. ${ }^{29}$ These boundaries are set by its nature and the acknowledgement of the counterpart as an autonomous being. Professionals may, for example, rightfully expect patients to show treatment adherence and honour the agreements made. ${ }^{21}$ Patients, on the other hand, can expect accountability for the advice given and continuing service and support from their health professionals. This dimension also includes refraining from revealing confidential information to others, as it appears to be paramount for autonomous agents to be in control of not only their physical body but also information about them and where or by whom it is processed. ${ }^{30}$ This applies especially to relationships in healthcare, as intimate and private information needs to be processed as part of this relationship and, thus, must be protected. ${ }^{29}$

Justice as a term has an, at least, threefold meaning. It is used in a basic non-comparable sense and as a formal or distributive comparable term entailing different rights and obligations. ${ }^{31}$ In its first sense, to be just means treating a person according to what is due to them, that is, what this person is entitled to have. ${ }^{32}$ This leads to a second and comparative meaning, because what is owed to another is based on morally relevant properties. From a formal point of view, equality in these properties requires equal treatment. However, what is owed to a person often depends on what is owed to others. In this third meaning, for example, under scarce resources, justice in this comparative sense is concerned with questions of fair distribution. Consequently, justice in the first sense is concerned with the overall quality of a therapeutic relationship, while the second and third sense are concerned with the quality of relationships compared with each other.

Humanity finally entails rights and duties to ensure that respect for individuality and being a person as integral part of the therapeutic relationship is ensured. Autonomy in the sense discussed above can only be expressed and exercised by the ability to shape one's own life according to one's own values and preferences. Exercising and acknowledging individuality as a result of this shaping and as a kind of uniqueness is, hence, deeply connected to the idea of autonomy. The dimension of humanity, thus, requires considering each other's uniqueness and individuality and treating each other with compassion. ${ }^{23} 24$

As a result, this moral framework allows both parties to be sure that their fundamental interests are protected. ${ }^{26}$ Both parties are able to show trustworthiness and to place trust in their counterpart, thus, laying out the foundations of a healing relationship. ${ }^{23} 24$ An overview of this framework can be seen in figure 1.

The special importance of trust and healing aspects in psychiatry has been stressed on different occasions. First, patient populations in psychiatric settings are particularly vulnerable, which results in different ethical problems compared with somatic settings. ${ }^{33} 34$ Patients' vulnerability is fed by disease patterns that can directly influence their ability to make autonomous choices and by external factors (eg, the dangers of stigmatisation associated with these diseases). ${ }^{34}$ This results in particular

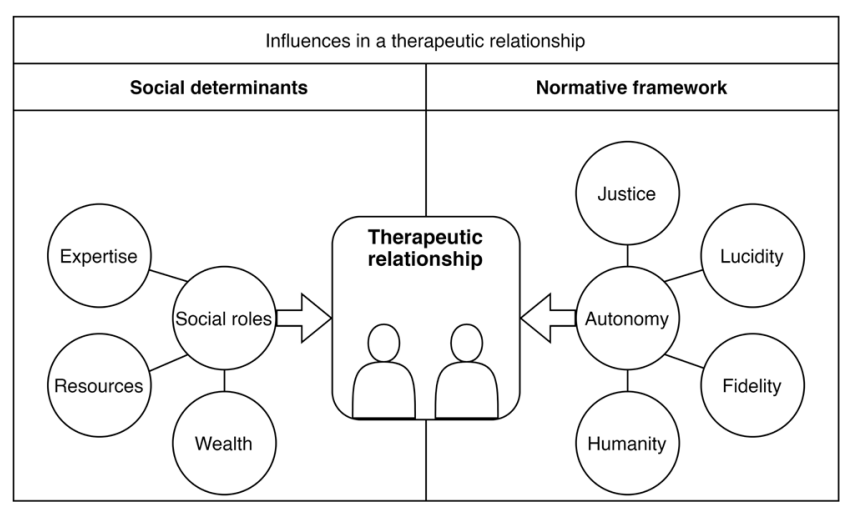

Figure 1 Social and normative dimensions of the therapeutic relationship in psychiatry. 
ethical problems that challenge the therapeutic relationship, for example, when decisions have to be made about the treatment of patients who are not fully capable of giving consent, or when therapist and patient happen to meet in a public space. Second, therapeutic relationships in psychiatry can be particularly private, personal and highly emotional. ${ }^{33}$ However, the most important difference compared with other settings is that the therapeutic relationship plays a more prominent role in therapeutic effectiveness. Depending on the type of therapy, the relationship is not a means to an end to protect important values but a treatment tool in itself. ${ }^{33}$ A strong and stable relationship can significantly improve the adherence to therapy and health outcomes. ${ }^{35}$ This requires particularly high standards between professionals and patients. ${ }^{36}$ The American Psychiatric Association, therefore, has noted in its comments on the ethical principles, as published by the American Medical Association, that a functioning relationship between professionals and patients is of paramount importance in psychiatry and awareness of the ethical problems connected is needed. ${ }^{36}$

It is important to stress that, as with every conceptual model, our approach is not able to capture a complete representation of all aspects of the therapeutic relationship, nor is it the only model that could be employed. However, it seems to be able to capture some very important assumptions which are required to investigate our initial concerns. First, it allows one to understand the therapeutic relationship against the background of social determinants. Starting from a two-sided encounter characterised by certain imbalances regarding power, it connects the social situation of the therapeutic relationship to the idea of moral requirements as a counterbalance. Second, it captures the plausible assumption that therapeutic relationships are not only relevant from an ethical perspective but also build the foundation for a healing relationship. Most of the obligations defined here can become signs of trustworthiness once they are exercised. The framework, thus, illustrates how both parties can be enabled to put trust in their counterpart, while their fundamental interests are protected. Third, by connecting the different layers, this model shows that changes in one of these layers would almost certainly have an effect on the others.

Against this background it is important to consider whether the therapeutic relationship can be changed by the use of videobased consultation. In order to shed more light on this, we elaborate below on empirical particularities in the communication between interlocutors in videoconferences and their influence on the therapeutic relationship in psychiatry.

\section{TECHNOLOGICAL MEDIATION OF THE THERAPEUTIC RELATIONSHIP IN VIDEO-BASED CONSULTATIONS}

We understand video-based consultation to be a technically mediated bidirectional communication over an undefined distance which combines optical and acoustic information. This is to adopt a (post)phenomenologically oriented perspective on the technology of video-based consultation. This view is defined by the attempt to analyse technology not based mainly on its intended functionality but in terms of the role it plays between humans and their world. ${ }^{37}$ Understanding video-based consultations as mediating is to focus on the question as to how technology interferes with the human perception of and experience with their reality. Mediating technologies in this understanding do not only transmit but transform what can be perceived, for example, by amplifying or reducing sensory information.

According to this, it has been suggested that the disruption of spatial coherence and mutual gaze and their 'reconnection' by technical means are unavoidable features of the experience of video-based mediation. ${ }^{38}{ }^{39}$ Following this thought, the transformation through technical reconnection may have an impact on many aspects of verbal and nonverbal communication between professionals and patients. ${ }^{13}$ To take a simple example, nonverbal communication especially might be particularly limited in videobased consultations and, at the same time, is often key to diagnosis and treatment. ${ }^{40}{ }^{41}$ Initial evidence for this hypothesis can be found in early works, such as O'Conaill et $a l,{ }^{42}$ who showed that the communication in videoconferences differs from the face-to-face situation independent of the transmission quality. Speakers give fewer backchannels and speaker-switching is more often initiated explicitly. The result is a generally more formal way of conducting a conversation. ${ }^{42}$ Furthermore, communication behaviour in groups has been shown to differ significantly in video-based settings. ${ }^{43}$ However, the analysis and investigation of therapeutic relationships in different psychiatric settings has been neglected and is mainly based on case studies yielding heterogeneous results. ${ }^{44}$ Notwithstanding this fact, studies outside the therapeutic area suggest that relationship factors are influenced by the videoconferencing setting. It has been shown, for example, that trust between participants in a videoconference can be quickly established, but is then more unstable than in a face-to-face setting. ${ }^{45}$ On the other hand, this effect can be mitigated if the conversation partners have met face-to-face previously or have time to discuss informal topics. ${ }^{46}$ Hence, it seems likely that such effects also have an impact on the therapeutic relationship in psychiatry in video-based consultations. In addition to affecting the communication, spatial disruption in technological mediation changes the social determinants between therapists and patients. As a result, it influences the asymmetry between both parties in the therapeutic relationship ${ }^{15} 47$ and, consequently, requires one to consider the adaptation of rights and duties.

We review the effects of technological mediation on therapeutic communication in common video-based consultations according to common-sense experience and existing empirical evidence. Based on the premise that videoconferencing creates a different lived experience of therapist-patient encounters mediated by a technical relay, we classify observable changes according to the four senses involved in communication: Vision, hearing, touch and physical proximity, and smell. In addition, we investigate aspects of spatial disruption and technical aspects of mediation as important components of this situation. An overview is given in figure 2 .

\section{Aspects of vision}

Video-based consultations usually allow only parts of the body to be seen. The image is, in most instances, reduced to face, shoulders, hands and parts of the torso. The body language cannot be perceived or is only perceived to a very limited extent. This concerns, for example, not only the sitting position and leg movements, but also gestures or trembling and cramped hands, self-touching and skipping actions as a sign of inner tension. Studies indicate that the perception of these body signals plays a more important role in the correct interpretation of strong emotions than that of facial expressions. ${ }^{48}$ In addition, the perception of details is often limited due to the reduction in size and lack of image resolution. This applies to parts of the clothing and details, such as the state of care of the fingernails or increased sweating, tears, pupil size or a tremor, which may not be perceptible. Colour shifts in the image transmission can make skin reactions, such as blushing or bleaching, tan or paleness, invisible. The importance of direct eye contact for recognising 


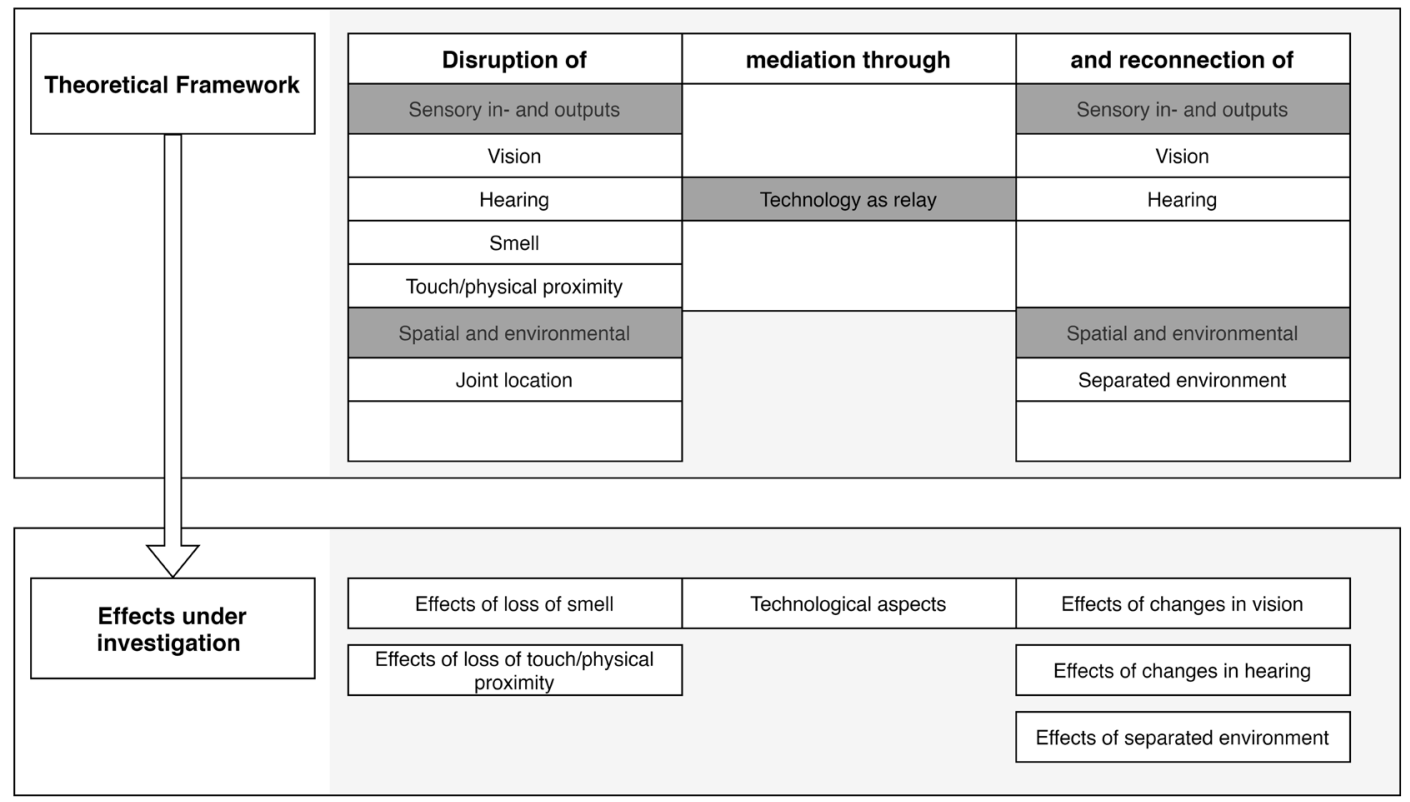

Figure 2 Aspects of technological mediation in video-based consultations.

emotions and building emotional connections has been widely studied. ${ }^{13}$ Direct eye contact in videoconferences is not possible, as the camera is mounted outside the screen, usually just above it, which can alternate the interaction. ${ }^{49}$ A software solution to this problem has already been worked on, but, so far, it has had no effect on practical use. ${ }^{50}$ Another visual feature of common software is that a miniature of one's own video is usually displayed on the screen, so that one can see not only one's counterpart but also oneself, as if in a small mirror. Studies show that this has significant effects on attention and communication behaviour. ${ }^{51}$

\section{Aspects of hearing}

Voice distortion, acoustic drop-outs and noise have to be taken into account when using videoconferencing due to persisting technical deficiencies in transmission. Intelligibility of speech and other verbal expressions, especially at low volume, is, thus, significantly decreased. Individual vocal characteristics and features of articulation can be overheard. In addition to distortions in transmission, the acoustic range of common built-in microphones is smaller than the natural range of the human voice, while their location is usually fixed. If a party speaks away from the microphone into the room, audibility is, thus, decreased. Using a headset, on the other hand, might circumvent these problems. However, to hear the voice of one's counterpart closer while speaking directly into the microphone creates a different experience than that of normal conversations. Since even inhaling, exhaling or sighing are transmitted, using a headset normally creates the impression of a closer physical distance and suggests an artificial intimacy. ${ }^{13}$ The transmission of background noise is another issue. Although it can be reduced by some software which intend to mimic the physiological 'cocktail party effect' and lift the voice of interest against the background, the effects can only partially compensate for the lack of directional hearing. ${ }^{52}$ Finally, there is a minimal time delay in the transmission of the audio signal in videoconferences, which is longer than in phone calls via a landline or mobile network. Studies have shown that this has an impact on the verbal interaction behaviour of conversation partners. ${ }^{42}$

\section{Aspects of touch and physical proximity}

Contrary to real conversation situations, there is no physical relationship between the parties in videoconferencing based on the perception of distance or alignment. Moving the chair away or turning towards the conversation partner, for example, is made more difficult by the relatively fixed sitting position in front of the screen. Spatial experiments used in some therapeutic settings, such as 'two-chair exercises,' can only be carried out in a modified form. By contrast, the visual proximity to each other is determined by the proximity to the camera, which can affect perception and behaviour. ${ }^{13}$ Depending on the position of the camera, the angle of view of the person opposite is changed. The camera on a laptop, for example, is usually mounted lower than eye level, giving the impression of looking at the counterpart from somewhere below. Whereas it is unusual in face-to-face consultations to sit frontally facing each other, this is the standard sitting position in videoconferences. Touching is obviously not possible in videoconferencing. This is advantageous for preventing infections and the physical protection of the conversation partners in highly tense situations. However, it is also not possible to shake hands, for example, for greetings, or to make connective gestures, such as handing out a handkerchief during the conversation. ${ }^{41}$ Since physical interaction with each other is not possible in videoconferencing, one party can be made a spectator of the other. What has already been frighteningly demonstrated in the live streaming of terrorist attacks in social media $^{53}$ can also be imagined for self-harm or suicide attempts in video-based consultations with a therapist.

\section{Aspects of smell}

The communicative significance of smell is rarely paid attention to. Nevertheless, the smell of conversational counterparts often influences our attitude towards them unconsciously. The conscious perception of the other person's odour is also important in the diagnostic and psychopathological assessment in psychiatry. The smell of perfume, alcohol, urine, working materials or lack of personal hygiene, for example, can be informative but cannot be perceived in videoconferencing. ${ }^{41}$ 


\section{Aspects of the environment}

One of the most interesting features of videoconferences is the complete loss of joint spatial location. This spatial disruption results in a very complex setting of two distinct environmental settings on each side of the encounter and two distinct virtual extensions of these locations which partly overlap.

First, already in advance of a video-based consultation, the environment of both parties is very different from that of a face-to-face meeting. In most cases, there are no approaches, leaving less time for preparation for the counterpart's arrival. This includes less time for mental and emotional preparation for the upcoming meeting if not consciously planned. Interaction starts when both parties are already sitting in front of the screen. It is not observable for either side what happened in the time immediately before the conversation began. In medical practice there is, for example, waiting time, greetings, orientation in the room, walking to the seating group, and choosing and taking a seat. A 'joint-attention,', that is, a common reference to a given exterior, is hardly possible in videoconferencing. It can, therefore, in a therapeutic setting, for example, be more difficult to jointly observe an aspect in the room, work together on a worksheet, look at graphic or therapy material together or have something demonstrated by the therapist. The influence of the environment on communication behaviour has not yet been sufficiently understood, although it is occasionally the subject of studies. ${ }^{54}$ The absence of physical copresence seems to make it more difficult to establish common ground, which can affect the performance of collaborating teams. ${ }^{46}$ Furthermore, it could be shown that even the mere knowledge that the conversation partner is far away reduces the primary willingness to trust and cooperate with them. ${ }^{55}$

Second, both parties of a videoconference find themselves situated in a different virtual environment. The videoconference usually takes place on a computer on which other programmes can also run and send messages in addition to the videoconferencing software, which can often be active in the background. The videoconferencing software itself, for example, can also indicate when the connection quality is low or an update is required. If the picture is not switched to full screen, other screen elements can distract the participants. Even the mere possibility of distraction through digital media can negatively influence the attention and empathy in the course of a conversation. ${ }^{13}$

Because both parties are situated in different environments, they are required to behave in two relatively separate contexts and their virtual extensions simultaneously, which can get complicated, for example, if the doorbell rings or someone else enters the room during a video call. If the videoconference takes place at home, the environment can convey a sense of security through familiarity, but also reduces the inner distance to everyday life. The lack of privacy in the domestic setting due to the potential proximity of household members can also be problematic for the therapeutic setting. ${ }^{56}$ On the other hand, objects of personal life, preliminary medical reports, one's own medication and therapy materials are more directly accessible and, thus, possibly simplify the exchange of information.

\section{Technical aspects}

As described above, a temporary deterioration of the transmission quality with a significant reduction of optical and acoustic resolution up to relevant connection disturbances are unfortunately still common. Since these usually occur suddenly and are of unforeseeable duration, this represents a significant disruption of the conversation. In the case of formal conversations, even the fear of such a disruption can lead to considerable stress.
This does not only lead to misunderstandings, but also possibly impairs the feeling of authenticity of the contact similar to the uncanny valley effect in robotics. ${ }^{57}$ In addition to these technical limitations, videoconferencing also offers the opportunity for a more intentional influence on the content of the transmission. One party, for example, can mute the other, terminate the connection abruptly, change their or the other party's background, voice or image, or, with the appropriate software, record and show a completely different video or audio. Furthermore, it is relatively easy to secretly record a video call or take pictures of the other party. This means, for example, that a technical instead of a physical threat could be realised. The conversation partner may no longer be physically attacked online but may be defamed in social media or rating portals, for example, or their computer may be hacked and data stolen.

\section{ETHICAL DIMENSIONS OF SHIFTS TOWARDS TECHNOLOGICAL MEDIATION THROUGH VIDEO-BASED CONSULTATIONS IN PSYCHIATRY}

It turns out that empirical studies and common sense experience against a (post)phenomenological background reveal a number of changes in communication between interlocutors in videoconferences, some of them far-reaching, as a result of different sensory, spatial and technical aspects of technological mediation. The question arises whether these changes in communication can be of ethical relevance when psychotherapy and psychiatric care is delivered online via video-based consultations.

Although there is evidence of various changes in communicational patterns triggered by the differences of communication situations experienced in videoconferencing, numerous empirical studies could find hardly any differences in the therapeutic relationship in video-based consultations compared with face-to-face contacts. In addition to clinical outcomes, patient and therapist satisfaction, subjective evaluation of the therapeutic relationship, or acceptance or adherence of patients are frequently examined. These findings are initially encouraging, but there are reasons to question the conclusion sometimes drawn that there is no relevant difference between video-based consultation and face-to-face therapy in psychotherapy. Patient satisfaction is undoubtedly a significant factor in the assessment of treatment success, but it has to be considered that patient satisfaction can be an uncertain parameter in clinical studies, since patients in most medical studies tend to evaluate their own treatment positively. ${ }^{58}$ It is interesting that therapists generally evaluate video-based consultation more critically..$^{59} \mathrm{~A}$ possible explanation might be that therapists are professionally trained to build and maintain a therapeutic relationship and are, therefore, more aware of it. ${ }^{17}$ It could be that subtle qualitative changes in the therapeutic relationship go largely unnoticed by patients, just as in other specialties, the professional quality of a medical treatment is not fully apparent to the patient and does not necessarily correlate with their stated treatment satisfaction. Acceptance and adherence can, furthermore, be positively biased by the fact that only relatively technology-oriented and technology-competent patients can be included in telepsychiatry studies.

Clinical outcomes as an index of the treatment quality of video-based consultations in psychiatry has been investigated in previous studies mostly using psychometric measures. However, even assuming that the treatment outcome of psychiatric care can be adequately measured by psychometric measurement, we think that this does not allow conclusions to be drawn regarding the overall quality of treatment. Since clinical outcomes are only one aspect of treatment quality, an overall assessment would 
have to consider additional factors including those related to the individual treatment process of patients according to their characteristics. Professionals and patients, for example, must interact and cooperate appropriately at all stages of treatment planning, procedure and evaluation in order to ensure that the therapy is ethically sound and oriented toward the well understood well-being of the patient. ${ }^{60}$ An impairment of the therapeutic relationship, as appears to be possible due to the changed communication in video-based consultations, is, in our understanding, of relevance regarding ethical considerations and an important factor for the quality of treatment concerning the process.

Based on a broader and ethically informed perspective, factors that affect the asymmetry between therapists and patients play a more important role and can be qualified in detail by focusing on the dimensions of the therapeutic relationship. In the following, therefore, we analyse possible influences of communicationchanging aspects of technological mediation in videoconferences on ethically relevant dimensions of the therapeutic relationship, according to the normative framework we introduced above. Hereby, we focus on the ethical dimensions of respect for autonomy, lucidity, fidelity and humanity as entailing rights and obligations that are mostly addressing the counterpart of a therapeutic encounter directly.

\section{Autonomy}

Effects to consider regarding questions of autonomy change particularly due to the effects of physical proximity and environmental aspects. Video-based consultation does not only distance parties from each other but introduces technology as a mediating relay position between both sides of the encounter. On the one hand, this enables the patient to exercise a greater amount of control over their side of the connection and to gain influence on more contextual factors compared with 'paying a visit' to their psychiatrist. In the latter case, for example, patients physically enter the highly structured medical sphere as help-seeking persons and have to comply with these structures and regulations on different levels. In video-based consultation, they are able to deliberately decide on the time, location and surroundings. ${ }^{61}$ This increase in autonomy in terms of control over the situation extends to the ability to influence the quality and quantity of information and information flow directly. Patients can decide how they are visually perceived, for example, through the adjustment of the camera angle, visibility of surrounding objects or brightness, and they can influence the audio transmission by technically adjusting the volume or tone of the signal. Most importantly, this kind of control in situations of physical contact is usually limited to one's own appearance, depending on the ability to control oneself and one's awareness of the situation. People in technically mediated situations, however, can-to a certain extent-shift this control and grant it to their counterparts, for example, by adjusting the size and position of the image on the screen, turning the volume up or down or even by silencing counterparts with a mouse click.

This gain of control over the determinants of the situations is counterbalanced by effects that also result from the relay position of the technological mediation. Regarding the former, control over one's own side of the connection might increase. However, ways to be aware of or control information once it passes the relay or influence what happens to this information at the other end are fairly limited. This includes being silenced or recorded without being aware of it and seeing and hearing only what is determined to be seen or heard by somebody else. In addition, technical risks of data breach or loss and misuse by third parties emerge from the relay itself which is usually controlled by neither of the parties, and have to be considered serious threats to the autonomy of both parties. As a result, the asymmetries between the patient and the therapist shift. While the possibility of self-determined control of context determinants increases, vulnerabilities are created at the level of informational control, which can no longer be clearly assigned to one of the two sides.

\section{Lucidity}

Shifts in asymmetries stemming from increasing abilities to control the context and information about oneself and decreasing awareness of how they are received have consequences for the requirements of lucidity between patients and therapists. Lucidity can no longer be understood as a byproduct of honest and truthful communication alone but must be connected to the duty of being a reliable sender of information who actively cares (by preparation, technical means and transparency) that information reaches its addressee. The same applies to technical interruptions, loss of information, cutoffs, drop-outs and vagueness in visual and acoustic information. Interruptions, loss of information and vagueness might be factors that regularly occur in conversations. In face-to-face situations, they are accounted for and minimised by a variety of implicit conversational practices or redundancy across different information channels. However, video-based consultation is characterised by the loss of additional information channels, while other information is vague or ambiguous to a greater extent. Shifts in control and awareness also increase the risk of information being channelled, shortened, filtered or misunderstood. Distortions in communication that may impact on the lucidity of the relationship have to be addressed more explicitly to be resolved. In addition, the reduced common reference space in the video-based consultation can impair the transfer of information by offering fewer opportunities for expression. This can be partially compensated for by technical means, such as the integration of photos and videos. However, this requires appropriate skills, which can be particularly problematic on the patient's side. It cannot be assumed that patients have the necessary resources to compensate for the disadvantages of video-based consultation at this point due to the particular vulnerability of psychiatric patients, especially regarding corresponding clinical conditions.

\section{Fidelity}

Two opposing observations are relevant for fidelity, understood as the commitment to a therapeutic relationship and continuing service and support. On the one hand, the empirical data show that ease of access to therapeutic services and consultations is facilitated because video-based consultation facilitates the bridging of spatial and temporal distances. ${ }^{62}{ }^{63}$ Furthermore, there is the possibility of maintaining a therapeutic relationship even when there is no physical proximity. On the other hand, it must be considered that technological mediation may create incentives for a rapid change in the relationship and a decrease in adherence. Relationships may be established more quickly but, by no means, more strongly.

Concerning questions of confidentiality, the use of videobased consultation poses special challenges connected to its environmental aspects. The agreement of confidentiality is usually connected to a physical location marking visible boundaries between an intimate and shared space where private information can be processed without reservation and an external space that explicitly carries the responsibility to refrain from any processing of this information. Knowing and agreeing to these 
boundaries and respecting the difference between those spaces is essential. However, this contained space in video-based consultation is fragmented through a technical relay, not locatable in a single space and boundaries are only partly visible.

This increases the risk of intentional or accidental breach of confidentiality because both sides lack the ability to enter, observe or control the respective remote space completely. It is impossible to know who is beneath or behind the camera, whether a door is open or closed, or family members are listening in the next room.

A second and even more troublesome question arises regarding the technical relay. All information passing it can, in principle, become subject of data loss, security breaches or leaks. In addition, perceiving the boundaries of the space of confidentiality requires technical competences of complex digital technologies to be able to assess which data is processed in what way and who has access. Risks regarding this technical dimension inevitably become part of the question of confidentiality. Hence, permeating the boundaries of physical space by remote relocation and reconnection by means of technological mediation fundamentally alters the nature of the confidentiality patients and professionals can and should agree on.

\section{Humanity}

Finally, the effects of the situation on duties of humanity must be considered. Duties of humanity are based on the necessity that interlocutors recognise each other as morally relevant and individual subjects and treat each other accordingly. This form of recognition requires a detailed understanding of each other and mutual empathy. While empathy is the result of making and maintaining contact, this level is not only influenced by the possibility and unpredictability of technical deficiencies but especially by inaccuracies and missing details, the lack of transmission of emotional auditory signals and the impossibility of making eye contact. Loss of detail and vagueness of information decreases the likelihood of successfully developing a detailed and empathic account of each other's individuality. ${ }^{9}$ As empirical evidence shows, this 'grainy resolution' of information and information loss tends to steer communication patterns towards a more formalised and prestructured conversation. ${ }^{42}$ In addition, building empathy requires attention above all else. As shown above, this can be significantly affected by changing environmental conditions and distracting factors in the virtual environment of video-based consultations. The technically mediated conversation, thus, increases the risk of failing to acknowledge each other's individuality and reduces communication to a less detailed but more error-proof standard regarding its transmission. This also means that the individuality of patients is less likely to be included in decisions concerning the diagnosis, treatment and planning of therapies.

\section{CONCLUSIONS AND OUTLOOK}

Video-based consultations in psychiatry will become a frequently used tool in the future. Use of this tool is one of the most important challenges for the development of psychiatry. ${ }^{19}$ This may apply to medical questions regarding treatment, diagnoses and outcome. However, as we have shown here, it also applies to the core of medical relationships. Seen from an ethical and empirical perspective, changes in the conversation situation between the therapist and the patient are extensive in video-based consultations compared with the face-to-face setting. Past studies do not seem to have investigated this sufficiently but focus primarily on psychometric outcome parameters, acceptance, adherence or satisfaction of patients and therapists in surveys when evaluating video-based consultations. Studies on communication via videoconferencing in other contexts, however, suggest that there are ethically relevant changes in the quality of conversations which may also affect the assessment of the general treatment quality from a medical perspective. These changes lead, on the one hand, to shifts in the area of patient autonomy and, on the other hand, have a great influence on obligations regarding lucidity, fidelity and humanity within the relationship between therapist and patient, hence requiring adjustments in rights and duties on both sides. Our observations show that these changes need to be carefully considered and practitioners need to be aware of these changes and adapt their behaviour accordingly.

We concede that there are limitations of our analysis. First, there may be other models of the therapeutic relationship that could be employed as a starting point. However, we do not want to contribute to the extensive debate on justificatory aspects of different models of this relationship. The model depicted here is merely used as a descriptive starting point for our analysis. In this sense, we deem it to be sufficiently specific regarding its ethical dimensions and broad enough to capture basic intuitions about the ethical behaviour between therapists and patients. This includes the respect for freedom of others, truth-telling, promise-keeping, avoiding of disparities and preserving of individuality. In this regard, it allows for a reconstruction of the important shifts of the therapeutic relationship. A second limitation applies to our methodology. We have not and did not intend to base our work on a systematic review of the existing literature. Hence, results should not be understood as a synthesis of existing evidence. This may limit the validity of our claims because we were not able to review all the existing evidence and indicates the necessity for further systematisation of empirical evidence. A third limitation to consider applies to the dimension of justice in the therapeutic relationship. We have not elaborated on this dimension for several reasons. The aim of this paper was to investigate the effects between patients and professionals. Justice, in this regard, has a less substantial meaning commonly understood as the overall quality or 'what we owe to each other'; we do not want to be concerned with this due to its overlap with all the other dimensions. However, we do not want to defend the claim that questions of justice in the second and third meaning outlined above are not important. We think that ethical questions regarding equal treatment and questions concerning a fair distribution of burdens need to be addressed. These include, for example, ethically problematic differences regarding access to digital technologies depending on social status or economic wealth. Doing justice to these difficult questions is, however, beyond the limits of this work.

To be able to assess these shifts more accurately, moreespecially qualitative-empirical studies are needed on the communicative characteristics of the therapeutic relationship in video-based consultations. This future research has to focus on the process rather than the outcome of the treatment. Methods for structured analyses of conversations and frameworks for the qualitative analysis of video material exist and might provide a suitable methodological approach to further investigate the effects. ${ }^{44}$ In a second step, special attention needs to be paid to the (post)phenomenological considerations on which our thoughts are based. Future research needs to aim at unveiling the perceptions and experiences of users with mediating technologies to fully understand the dynamics of mediation. In this endeavour to understand how technology shapes perception and choices, Aagaard has, for example, suggested focusing on (1) in-depth exploration of typical use and (2) critical comparison 
of multiple versions of a technology. ${ }^{64}$ While the first is suitable to generate in-depth knowledge and explore the bandwidth of experiences in video-based consultation, the latter may provide the opportunity to generalise such knowledge by abstracting from a specific version of a technology. A challenge remains concerning integrating such approaches into a normative framework of analysis. Although healthcare technologies are rapidly evolving and a growing need for empirically informed normative frameworks is evident, only a very few approaches for such an empirically informed ethics of healthcare technologies exist. However, such frameworks can help to focus empirical research on features that concern ethically relevant aspects of the quality of the relationship, such as the compensation of incongruities in the therapeutic relationship, vagueness in communication and loss of information.

In addition, we suggest that steps towards the further implementation and provision of video systems for video-based consultation (eg, implementation in hospitals) should include considerations regarding ethical aspects. This may not only mean to be aware of such effects but also to engage in measures on the structural level to mitigate negative effects as far as possible. Measures to this end could include the provision of a stable internet connection, optimised hardware and software, and special rooms and environmental conditions for conducting online video therapy in hospitals. The development of technologies for video-based consultations should involve therapists and patients and take into account research findings on the communicative level. Fading out the miniature of one's own image, for example, and the technical correction possibilities of the visual axis to enable eye contact should be discussed. The functionality of software should focus on optimising the quality of the conversation in psychiatric settings, taking into account mediumspecific features and differences to the face-to-face setting.

We, finally, conclude that therapists and patients should be better trained in the appropriate and communication-enhancing use of video-based consultation. As far as possible, all participants should know the communicative characteristics, chances and risks of this form of therapy and be informed about possibilities to improve the therapeutic setting in video-based consultation. Corresponding training offers have so far been rare and insufficiently supported by employers and health insurance companies. In the course of the pandemic, many clinics and therapists, out of sheer necessity, simply took the next available software and hardware, set it up in their office and started video-based consultation for patients right away. This has worked surprisingly well and is now to be continued in the long term. ${ }^{65}$ However, the experience gained in the meantime does not replace the critical reflection and ethical aspects of the use of this technology in therapy. Procedures which have already been established should, therefore, be revised.

As long as it has not been clarified how the use of video-based consultation in psychiatry is to be evaluated ethically and as long as the shortcomings in its application mentioned here remain, we advocate a carefully limited and cautious use of this form of therapy. In our opinion, for ethical reasons, it should currently only be used where and when face-to-face therapy is not possible and risks of treatment delay clearly outweigh the risk of the digitised setting. As far as possible, video-based consultation should be considered as a supplement to face-to-face contacts, avoiding settings in which therapists and patients have not met each other in person before. In all cases, application of video-based consultations in psychiatry require the awareness of possible downsides and knowledge of empirical research results on effects on communication to avoid those pitfalls described above. We understand this awareness to be a duty of medical ethicists in research and therapists in clinical work emerging from the idea of ethical relationships in medicine itself, which requires practitioners to take a position of caution and put the relationship between patients and professionals at the centre of care.

\section{Note}

This article was written during the COVID-19 pandemic and was worked out in online videoconferences by the authors who had never met personally until the submission.

Contributors The authors contributed equally.

Funding The authors have not declared a specific grant for this research from any funding agency in the public, commercial or not-for-profit sectors.

Competing interests None declared.

Patient consent for publication Not applicable.

Provenance and peer review Not commissioned; externally peer reviewed.

There are no data in this work.

\section{REFERENCES}

1 Drago A, Winding TN, Antypa N. Videoconferencing in psychiatry, a meta-analysis of assessment and treatment. Eur Psychiatry 2016;36:29-37.

2 Hariman K, Ventriglio A, Bhugra D. The future of digital psychiatry. Curr Psychiatry Rep 2019;21(9):88.

3 Stoll J, Sadler JZ, Trachsel M. The ethical use of Telepsychiatry in the Covid-19 pandemic. Front Psychiatry 2020;11:665.

4 Deslich S, Stec B, Tomblin S, et al. Telepsychiatry in the 21(st) century: transforming healthcare with technology. Perspect Health Inf Manag 2013;10.

5 Turvey C, Coleman M, Dennison 0, et al. Ata practice guidelines for video-based online mental health services. Telemed J E Health 2013;19(9):722-30.

6 Stoll J, Müller JA, Trachsel M. Ethical issues in online psychotherapy: a narrative review. Front Psychiatry 2019;10:993.

7 Hubley S, Lynch SB, Schneck C, et al. Review of key telepsychiatry outcomes. World J Psychiatry 2016:6(2):269-82.

8 Shore $\mathrm{JH}$. Telepsychiatry: videoconferencing in the delivery of psychiatric care. Am J Psychiatry 2013;170(3):256-62.

9 van Wynsberghe A, Gastmans C. Telepsychiatry and the meaning of in-person contact: a preliminary ethical appraisal. Med Health Care Philos 2009;12(4):469-76.

10 Norman S. The use of telemedicine in psychiatry. J Psychiatr Ment Health Nurs 2006;13(6):771-7.

11 Jenkins-Guarnieri MA, Pruitt LD, Luxton DD, et al. Patient perceptions of Telemental health: systematic review of direct comparisons to In-Person psychotherapeutic treatments. Telemed J E Health 2015:21(8):652-60.

12 Chakrabarti $S$. Usefulness of telepsychiatry: a critical evaluation of videoconferencingbased approaches. World J Psychiatry 2015:5(3):286-304

13 Russell Gl. Screen relations the limits of Computer-Mediated psychoanalysis and psychotherapy. Taylor \& Francis Ltd, 2018.

14 Scharff JS, ed. Psychoanalysis online: Mental health, teletherapy and training. London: Karnac, 2013.

15 Miller EA. Telemedicine and doctor-patient communication: a theoretical framework for evaluation. J Telemed Telecare 2002;8(6):311-8.

16 Del Re AC, Flückiger $C$, Horvath AO, et al. Examining therapist effects in the alliance-outcome relationship: a multilevel meta-analysis. J Consult Clin Psychol 2021;89(5):371-378.

17 Lopez A, Schwenk S, Schneck CD, et al. Technology-Based mental health treatment and the impact on the therapeutic alliance. Curr Psychiatry Rep 2019:21(8):76.

18 Brody H. The physician-patient relationship: models and criticisms. Theor Med 1987;8(2):205-20.

19 Koch U, Cratsley K, et al. The History and Ethics of the Therapeutic Relationship. In: Trachsel M, Gaab J, Biller-Andorno N, eds. The Oxford Handbook of psychotherapy ethics. Oxford University Press, 2020.

20 Stirrat GM, Gill R. Autonomy in medical ethics after O’Neill. J Med Ethics 2005;31(3):127-30.

21 Goodyear-Smith F, Buetow S. Power issues in the doctor-patient relationship. Health Care Anal 2001;9(4):449-62.

22 Kalliainen LK, Lichtman DM. Current issues in the physician-patient relationship. $J$ Hand Surg Am 2010;35(12):2126-9.

23 Brody H. The Physician-Patient-Relationship. In: Veatch RM, ed. Medical ethics. 2 edn. Sudbury, Mass: Jones and Bartlett Publ, 1997: 75-102.

24 Marcum JA. Humanizing modern medicine. Dordrecht: Springer Netherlands, 2008.

25 Fried C. Medical experimentation: personal integrity and social policy. New York: Oxford University Press, 2016.

26 O'Neill O. Autonomy and trust in bioethics. Cambridge: Cambridge Univ. Press, 2007.

27 Childress JF. The place of autonomy in bioethics. Hastings Cent Rep 1990;20(1):12. 
28 Beauchamp TL, Childress JF. Principles of biomedical ethics. 5 edn. Oxford: Oxford Univ. Press, 2001.

29 Kitchener KS. Intuition, critical evaluation and ethical principles: the foundation for ethical decisions in counseling psychology. Couns Psychol 1984;12(3):43-55.

30 O'Brien J, Chantler C. Confidentiality and the duties of care. J Med Ethics 2003;29(1):36-40.

31 Buchanan A. Mathieu D. Philosophy and Justice. In: Cohen RL, ed. Justice: views from the social sciences. New York: Springer Science + Business Media, 1986: 11-45.

32 Gillon R. Justice and medical ethics. Br Med J 1985;291(6489):201-2.

33 Radden J. Notes towards a professional ethics for psychiatry. Aust N Z J Psychiatry 2002;36(1):52-9.

34 Radden J. Psychiatric ethics. Bioethics 2002;16(5):397-411.

35 Krupnick JL, Sotsky SM, Elkin I, et al. The role of the therapeutic alliance in psychotherapy and pharmacotherapy outcome: findings in the National Institute of mental health treatment of depression Collaborative research program. Focus 2006;4(2):269-77.

36 American Psychiatric Association. The principles of medical ethics with annotations especially applicable to psychiatry, 2013.

37 Verbeek P-P. Materializing morality. Sci Technol Human Values 2006;31(3):361-80.

38 Friesen N. Telepresence and Tele-absence: A Phenomenology of the (In)visible AlienOnline. P\&P 2014:8(1):17-31.

39 Ferencz-Flatz C. Ten theses on the reality of video-chat: a phenomenological account. Communications 2019:44(2):204-24.

40 Foley GN, Gentile JP. Nonverbal communication in psychotherapy. Psychiatry 2010;7(6):38-44.

41 Miller EA. The technical and interpersonal aspects of telemedicine: effects on doctorpatient communication. J Telemed Telecare 2003;9(1):1-7.

42 O'Conaill B, Whittaker S, Wilbur S. Conversations over video conferences: an evaluation of the spoken aspects of Video-Mediated communication. HumanComputer Interaction 1993;8(4):389-428.

43 Sellen A. Remote conversations: the effects of mediating talk with technology. Hum Comput Interact 1995;10(4):401-44.

44 Miller EA. Telemedicine and doctor-patient communication: an analytical survey of the literature. J Telemed Telecare 2001:7(1):1-17.

45 Bos N, Olson J, Gergle D. Effects of four computer-mediated communications channels on trust development. In: Wixon D, ed. Proceedings of the SIGCHI conference on Human factors in computing systems Changing our world, changing ourselves - $\mathrm{CHI}$ '02. 135. New York, New York, USA: ACM Press, 2002.

46 Olson JS, Olson GM. Bridging Distance: Empirical Studies of Distributed Teams. In: Galletta DF, Zhang Y, eds. Human-computer interaction and management information systems. Applications. London: Routledge, 2006: II. 27-30.

47 Agha Z, Roter DL, Schapira RM. An evaluation of patient-physician communication style during telemedicine consultations. J Med Internet Res 2009;11(3):e36.
48 Aviezer H, Trope Y, Todorov A. Body cues, not facial expressions, discriminate between intense positive and negative emotions. Science 2012:338(6111):1225-9.

49 Tran M, Sen T, Haut K. Are you really looking at me? A Feature-Extraction framework for estimating interpersonal eye gaze from conventional video, 2019. Available: https://arxiv.org/pdf/1906.12175

50 Isikdogan LF, Gerasimow T, Michael G. Eye Contact Correction using Deep Neural Networks. In: 2020 IEEE winter conference on applications of computer vision (WACV. IEEE, 2020: 3307-15.

51 Miller MK, Mandryk RL, Birk MV. Through the Looking Glass. In: Mark G, Fussell S, Lampe C, eds. Proceedings of the 2017 chi conference on human factors in computing systems. New York, NY, USA: ACM, 2017: 5271-83.

52 Bronkhorst AW. The cocktail Party effect: research and applications. J Acoust Soc Am 1999:105(2):1150.

53 Conway M, Dillon J. Future trends: Live-Streaming terrorist attacks? VOX-Pol.

54 Whittaker S. Things to talk about when talking about things. Human-Computer Interaction 2003;18(1-2):149-70.

55 Bradner E, Mark G. Why distance matters. In: Churchill EF, McCarthy J, Neuwirth C, eds. Proceedings of the 2002 ACM conference on Computer supported cooperative work - CSCW '02. 226. New York, New York, USA: ACM Press, 2002.

56 Gottlieb L. In psychotherapy, the toilet has become the new couch, 2020. Available: https://www.nytimes.com/2020/04/30/opinion/psychotherapy-remote-covid.html [Accessed 02 Oct 2020].

57 Dettbarn I. Skype as the uncanny third. In: Scharff JS, ed. Psychoanalysis online: mental health, teletherapy and training. London: Karnac, 2013: 15-25.

58 Hall JA, Dornan MC. Meta-Analysis of satisfaction with medical care: description of research domain and analysis of overall satisfaction levels. Soc Sci Med 1988;27(6):637-44.

59 Cowan KE, McKean AJ, Gentry MT, et al. Barriers to use of Telepsychiatry: clinicians as gatekeepers. Mayo Clin Proc 2019;94(12):2510-23.

60 Egger JW. Zuerst heile MIT dem Wort...? Zur Bedeutung Der Kommunikation in Der Humanmedizin. Psychologische Medizin 2012;23(1):38-49.

61 Chester A, Glass CA. Online counselling: a descriptive analysis of therapy services on the Internet. Br J Guid Counc 2006;34(2):145-60.

62 Wodarski J, Frimpong J. Application of E-Therapy programs to the social work practice J Hum Behav Soc Environ 2013;23(1):29-36.

63 Cipolletta S, Mocellin D. Online counseling: An exploratory survey of Italian psychologists' attitudes towards new ways of interaction. Psychother Res 2018;28(6):909-24.

64 Aagaard J. Introducing postphenomenological research: a brief and selective sketch of phenomenological research methods. Int J Qual Stud Educ 2017;30(6):519-33.

65 Feijt M, de Kort Y, Bongers I, et al. Mental health care goes online: practitioners experiences of providing mental health care during the COVID-19 pandemic. Cyberpsychol Behav Soc Netw 2020;23(12):860-4. 